Strapline: Extracellular vesicles

\title{
Diffusion and transport of extracellular vesicles
}

Cell-derived extracellular vesicles are important intercellular communicators involved in many biological processes and diseases, including cancer and cardiovascular diseases, but thus far, it was poorly understood how they navigate within complex extracellular matrices.

\section{Gregor Fuhrmann}

Extracellular vesicles (EVs) have attracted tremendous attention in the past years - not only their fundamental biology but also their potential application for therapeutic avenues ${ }^{1}$. EVs are nanoparticles shed by almost all cell types in vitro and in vivo. They are composed of a phospholipid bilayer that incorporates several distinct surface and membrane proteins. In addition, EVs can carry cargoes including miRNA, proteins or resistance genes. It has been proposed that EVs can selectively and specifically transfer their biological information between donor and recipient cells, both in proximity and at distance ${ }^{2}$. The ability of EVs to protect and deliver their information has sparked substantial research interest to understand basic cell communication mechanisms and to potentially use them for therapeutic applications. Although these mechanisms of EV targeting and entry into target cells are not yet completely understood ${ }^{3}$, there are several examples in the literature evidencing that these natural carriers could be used for the delivery of compounds to treat various dispositions $s^{4,5}$.

Interestingly, EV characterisation often focusses mainly on particle size and number, detection of specific surface proteins and nucleic acid cargo. Indeed, the role of the mechanical properties of EVs and the impact of transport mechanisms on interactions with complex cellular environments has been poorly investigated. Writing in Nature Nanotechnology, Lenzini et al. now used model hydrogels to reveal the diffusion and transportation processes that EVs undergo in extracellular matrices ${ }^{6}$ (ECM) (Figure 1). The authors hypothesised that, in order to avoid ECM accumulation, which could limit the efficiency of EVs to reach their target cells, there is a matrix-dependent mechanism of vesicle clearance and transport. Using mesenchymal stem cell (MSC)-derived EVs and alginate hydrogels, EV transport in confined environments was studied in comparison to other nanocarriers. MSC EVs were selected because they can serve as low-immunogenicity drug carriers ${ }^{7}$ and they are often present in interstitial matrix regions. Alginate represents a simple and widely applicable model for nondegradable hydrogels that mimic the mechanical properties of decellularised ECM.

In an initial screening in alginate gels, the researchers observed that small dextran molecules were released within $24 \mathrm{~h}$ of incubation while larger polystyrene beads were trapped within the hydrogel matrix. By contrast, EVs were able to escape stress relaxing hydrogels to a higher extent than the artificial polystyrene beads, despite having similar particle sizes. Interestingly, phospholipid carriers, such as liposomes, of the same size were not released to a higher extent than EVs, indicating that the effect was vesicle-specific and dependent on matrix mechanics. It was thus hypothesised that the presence of the lipid membrane alone is not sufficient to enhance EV transport, but that surface proteins may also be involved in this phenomenon.

To better understand the mechanosensitivity of EV release from hydrogels, the researchers employed high-speed 3D microscopy with deconvolution to visualise and quantify EV transport at different conditions and over time. This 3D approach was combined with the calculation of the specific cage size that EVs need to overcome to freely transport within the matrix. These 'cages' can be considered as cavities in the hydrogel, which display a defined size and must be crossed for entrapped particles to transport through the matrix. Interestingly, EVs in stiff stress-relaxing matrices escape the cages more rapidly than EVs in soft stress-relaxing matrices. In addition, stiff elastic moduli prevent EVs from escaping hydrogel cages, highlighting the fact that matrix stress relaxation is crucial for EV escape out of confined matrices.

Finally, the impact on water-transporting aquaporins in the EV membrane was studied. The researchers suspected that water flux through these channels may influence mechanical EV properties. 
Indeed, the presence of functional aquaporins in EVs is required for water permeation, which in turn makes EVs deformable. Knockdown of aquaporins in MSCs led to the production of EVs which showed a decreased release from hydrogels. Lyophilisation of EVs - which may influence membrane integrity $^{8}$ and aquaporin functionality - also caused loss of EV transport in hydrogels, which indicates an important role of water permeation for EV deformability and navigation within an ECM.

The work by Lenzini et al. adds important information to our understanding of fundamental EV communication processes and the physiological role of EVs within biological matrices, and thus, is a significant advance in the field. The mechanical behaviour of EVs in complex environments is also relevant for their application as drug carriers, for example, to understand EV behaviour under shear stress in the blood circulation following systemic administration. Indeed, for liposomes, it was shown that the mechanical properties are important factors during cell interaction and that elasticity of nanoparticles influences their uptake in cancer cells ${ }^{9}$. In the EV field, mechanical moduli are still insufficiently studied. For erythrocyte-derived EVs, membrane fluidity is influenced by the disease state of the patient ${ }^{10}$, which may be instrumental information for devising new diagnostics. In addition, the results presented in this work may also shine light on the impact of exogenously integrated water channels on the behaviour of artificial liposomes. Modulating the deformability of these phospholipid carriers may help their tissue distribution and delivery efficiency ${ }^{11}$.

Figure 1. Transport mechanisms of extracellular vesicles (EVs) in model hydrogels. Alginate hydrogels were used as model for extracellular matrix to investigate the transport mechanisms of EVs. Dependency on mechanical moduli of the hydrogel and cage sizes were assessed by high-speed 3D microscopy. 
Gregor Fuhrmann is at the Helmholtz Institute for Pharmaceutical Research Saarland, Saarbrïcken, Germany. gregor.fuhrmann@helmholtz-hzi.de

\section{References}

1 Fuhrmann, G. et al. Nano Today 10, 397-409 (2015).

2 Meldolesi, J. Curr Biol 28, R435-r444 (2018).

3 Thery, C. et al. Nat. Rev. Immunol. 2, 569-579 (2002).

$4 \quad$ El Andaloussi, S. et al. Nat Rev Drug Discov 12, 347-357 (2013).

$5 \quad$ Schulz, E. et al. J. Control. Release 290, 46-55 (2018).

6 Lenzini, S. et al. Nat. Nanotechnol. (2020).

7 Gimona, M. et al. Int. J. Mol. Sci. 18, 1190 (2017).

$8 \quad$ Frank, J. et al. Sci Rep 8, 12377 (2018).

9 Guo, P. et al. Nat Comm 9, 130 (2018).

10 Vorselen, D. et al. Nat Comm 9, 4960 (2018).

11 Holme, M. N. et al. Nat Nano 7, 536-543 (2012). 